\title{
Evaluation of six minute walking test in patients with single chamber rate responsive pacemakers
}

\author{
Frank Provenier, Luc Jordaens
}

\begin{abstract}
Objective-To validate a simplified exercise protocol (the six minute walk) as a means of evaluating pacing modes and rate responsive pacemakers.

Design-Two groups of patients with different pacemaker types (activity and dual sensor) were randomly assigned to four consecutive pacing settings (fixed rateor VVI at 60,85 , and $110 / \mathrm{min}$, and optimal rate response-or VVIR). A third group of elderly patients without arrhythmias or conduction disturbances formed a control population.

Setting-Ambulatory consultation for patients with a pacemaker in a tertiary referral centre for treatment of arrhythmias.

Subjects-16 patients with rate responsive pacemakers for complete heart block and limited functional capacity and 13 controls with normal chronotropic competence.
\end{abstract}

Interventions-Submaximal exercise protocel with 6 minutes walking and continuous recording of electrocardiogram.

Main outcome measures-Achieved distance and scored degree of exertion during walking in the four settings in the patients with a pacemaker; differences in rate behaviour in VVIR mode between the two pacemaker types; comparison of the pacing rate with the heart rate of the control population.

Results-The six minute walk was performed better in VVIR than VVI 60 . In VVI 85 the distance was also significantly longer than in VVI 60 . The rise in pacing rate of activity pacemakers was steeper than that of the dual sensor pacemakers and differed from the heart rate in the controls at 90 seconds.

Conclusions-The studied test protocol was able to show differences in exercise capacity between pacing modes. Different rate responses between the evaluated sensor types could be established. The six minute walking test gives enough information to program and reprogram single chamber rate responsive pacemakers.

Dr L Jordaens, Department of Cardiology, University Hospital, De Pintelaan 185, B-9000 Ghent, Belgium.

Accepted for publication 26 January 1994
Optimal programming of pacing variables is important in rate adaptive pacemakers to improve exercise capacity and reduce symptoms. ${ }^{12}$ Individual programming should be adapted to the age of patients and their associated medical conditions, as well as to functional capacity. ${ }^{34}$ Because evaluation with standardised tests such as treadmill and bicycle ergometry is time consuming and not always feasible in patients with pacemakers (as they are often elderly, with physical limitations), rate responsive devices often remain programmed in VVI or in the nominal settings of rate response. ${ }^{56}$

We looked for a simple, short, safe, but objective, test to help programme rate modulating pacemakers. Such a test should require only a minimum of special technical equipment. Furthermore, a good relation to everyday activities and good reproducibility are desirable. The exercise protocol should be feasible for evaluating different types of pacemakers, and elderly patients should feel comfortable about doing the test. We report our evaluation of walking for six minutes as an alternative exercise test in a study group of patients with single chamber rate responsive pacemakers. The accuracy of the rate behaviour of the different sensors was compared with the rate augmentation in control subjects.

\section{Patients and methods}

PATIENTS

A total of 32 patients were entered in the study. Thirteen patients without arrhythmias or conduction disturbances formed the control population. Age ranged from 58 to 86 years (mean 72). Five patients had ischaemic heart disease, one patient had congestive cardiomyopathy, and another had a valve prosthesis. The functional capacity was limited in 11 patients (nine with class 2 disease and two with class 3 disease according to the New York Heart Association classification).

Of the 19 patients with pacemakers, three were excluded because atrioventricular conduction reappeared during the exercise protocol. The remaining 16 patients (12 women and four men) showed pacemaker dependency (table). They were aged between 52 and 74 years (mean 66, not significantly different from the control group). The indication 
Data on 16 patients with pacemakers who underwent six minute walking test

\begin{tabular}{|c|c|c|c|c|c|c|c|c|}
\hline $\begin{array}{l}\text { Case } \\
\text { No }\end{array}$ & $\begin{array}{l}\text { Age } \\
\text { (years) }\end{array}$ & $\operatorname{Sex}$ & Heart disease & Atrial arrhythmia & NYHA class & $\begin{array}{l}\text { Ejection } \\
\text { fraction (\%) }\end{array}$ & $\begin{array}{l}\text { Pacemaker } \\
\text { indication }\end{array}$ & $\begin{array}{l}\text { Pacemaker } \\
\text { type }\end{array}$ \\
\hline $\begin{array}{l}1 \\
2 \\
3\end{array}$ & $\begin{array}{l}74 \\
52 \\
69\end{array}$ & $\begin{array}{l}F \\
F \\
F\end{array}$ & $\begin{array}{l}\text { Dilated cardiomyopathy } \\
\text { Ischaemic heart disease } \\
\text { Ischaemic heart disease, mitral } \\
\text { valve replacement }\end{array}$ & $\begin{array}{l}\text { CAF } \\
\text { PAF } \\
\text { CAF }\end{array}$ & $\begin{array}{l}3 \\
3 \\
2\end{array}$ & 62 & $\begin{array}{l}\text { Ablation of His bundle } \\
\text { Ablation of His bundle } \\
\text { Ablation of His bundle }\end{array}$ & $\begin{array}{l}\text { Topaz } \\
\text { Excel } \\
\text { Excel }\end{array}$ \\
\hline 4 & 66 & $\mathbf{F}$ & $\begin{array}{l}\text { Hypertrophic cardiomyopathy, } \\
\text { aortic valve replacement, } \\
\text { ventricular tachycardia }\end{array}$ & Atrial flutter & 2 & 57 & Ablation of His bundle & Excel \\
\hline $\begin{array}{l}5 \\
6 \\
7 \\
8\end{array}$ & $\begin{array}{l}65 \\
70 \\
72 \\
54\end{array}$ & $\begin{array}{l}\mathbf{F} \\
\mathbf{M} \\
\mathbf{M} \\
\mathbf{M}\end{array}$ & $\begin{array}{l}\text { Mitral valve replacement } \\
\text { Ventricular septum defect } \\
\text { Ischaemic heart disease } \\
\text { Atrial septum defect, dilated } \\
\text { cardiomyopathy }\end{array}$ & $\begin{array}{l}\text { CAF } \\
\text { Atrial flutter } \\
\text { PAF } \\
\text { CAF }\end{array}$ & $\begin{array}{l}2 \\
1 \\
2 \\
2\end{array}$ & $\begin{array}{l}53 \\
34 \\
52 \\
35\end{array}$ & $\begin{array}{l}\text { Ablation of His bundle } \\
\text { Complete heart block } \\
\text { Ablation of His bundle } \\
\text { Ablation of His bundle }\end{array}$ & $\begin{array}{l}\text { Excel } \\
\text { Excel } \\
\text { Topaz } \\
\text { Topaz }\end{array}$ \\
\hline 9 & 69 & $\mathbf{F}$ & $\begin{array}{l}\text { Ischaemic heart disease, } \\
\text { torsades de pointes }\end{array}$ & CAF & 2 & 68 & Ablation of His bundle & Topaz \\
\hline $\begin{array}{l}10 \\
11 \\
12\end{array}$ & $\begin{array}{l}70 \\
72 \\
66\end{array}$ & $\begin{array}{l}\mathbf{F} \\
\mathbf{F} \\
\mathbf{F}\end{array}$ & $\begin{array}{l}\text { Ischaemic heart disease } \\
\text { None } \\
\text { None }\end{array}$ & $\begin{array}{l}\text { PAF } \\
\text { CAF } \\
\text { PAF }\end{array}$ & $\begin{array}{l}1 \\
2 \\
2\end{array}$ & $\begin{array}{l}85 \\
53 \\
44\end{array}$ & $\begin{array}{l}\text { Complete heart block } \\
\text { Ablation of His bundle } \\
\text { Sick sinus syndrome plus } \\
\text { ablation of His bundle }\end{array}$ & $\begin{array}{l}\text { Topaz } \\
\text { Topaz } \\
\text { Synchrony }\end{array}$ \\
\hline 13 & 72 & $\mathbf{F}$ & None & Atrial flutter & 2 & 61 & $\begin{array}{l}\text { Sick sinus syndrome plus } \\
\text { ablation of His bundle }\end{array}$ & Topaz \\
\hline $\begin{array}{l}14 \\
15\end{array}$ & $\begin{array}{l}62 \\
64\end{array}$ & $\begin{array}{l}\mathrm{M} \\
\mathrm{F}\end{array}$ & $\begin{array}{l}\text { Ischaemic heart disease } \\
\text { Atrial, mitral, and tricuspid } \\
\text { valve replacement }\end{array}$ & $\begin{array}{l}\text { CAF } \\
\text { CAF }\end{array}$ & $\begin{array}{l}3 \\
2\end{array}$ & 24 & $\begin{array}{l}\text { Ablation of His bundle } \\
\text { Postsurgical complete } \\
\text { heart block }\end{array}$ & $\begin{array}{l}\text { Topaz } \\
\text { Legend }\end{array}$ \\
\hline 16 & 59 & $\mathbf{F}$ & None & CAF & 2 & & Ablation of His bundle & Legend plus \\
\hline
\end{tabular}

NYHA, functional classification of the New York Heart Association; CAF, chronic atrial fibrillation; PAF, paroxysmal atrial fibrillation.

for ventricular pacing was complete heart block, spontaneous or surgical, in three patients and after radiofrequency ablation of the His bundle in 13 patients (because of disabling atrial fibrillation in 11 and because of the sick sinus syndrome in two). One patient with ischaemic heart disease had torsades de pointes during quinidine treatment. Chronic atrial fibrillation was diagnosed in nine patients; in four the arrhythmia was paroxysmal. Three patients showed atrial flutter. Organic heart disease was present in 12 patients, of whom six had ischaemic heart disease. Four had had valve replacement, and two were followed up after correction of a septal defect. One patient had hypertrophic cardiomyopathy, and an implantable cardioverter defibrillator for ventricular tachycardia. Only two patients had class 1 disease. The others showed restrictions in physical capacity: 11 had class 2 disease and three class 3 disease. In 12 patients the left ventricular ejection fraction was evaluated by technetium scintigraphy and ranged from $24 \%$ to $85 \%$ (mean $52 \%)$. All pacemakers were implanted in the left pectoral site, with a lead stimulating the right ventricle. An activity sensing device was implanted in seven patients; five of them used an accelerometer (Excel, CPI), and two a piezoelectric crystal (Legend, Medtronic, and Synchrony, Siemens-Pacesetter). The patient with the DDDR device (Synchrony) had it reprogrammed to VVIR when atrial fibrillation persisted. The remaining nine patients received dual sensor pacemakers: eight Topaz (activity with QT interval, Vitatron) and one Legend plus (activity and minute ventilation, Medtronic) models.

\section{Methods}

The six minute walking test was performed in a corridor $45 \mathrm{~m}$ long with $1 \mathrm{~m}$ and $5 \mathrm{~m}$ marks on the floor. For this protocol direct cardiac monitoring was performed to recognise serious arrhythmias during the test. The patients carried a two channel Holter recorder, to measure the heart rate (controls) or pacing rate (patients with pacemakers), the QT interval, and the occurrence of arrhythmias. During the exercise stimulation was moderate so that the patient could walk as far as possible. The patients had no knowledge about how much time had elapsed during the walk. They wore allowed to use aids such as a walking stick during the walk if they habitually used one. The distance walked was recorded directly after the six minute walk. In the recuperation period the patients were asked to score the workload of the test, based on the non-linear Borg scale.?

The patients with pacemakers walked the first time in nominal-that is, the mode recommended by the manufacturer-VVIR setting to adapt to this form of exercise. This walk was not included in further analysis. Afterwards; they were randomly assigned to walk again with four different settings: VVI with three different pacing rates $(60 / \mathrm{min}$, $85 / \mathrm{min}, 110 / \mathrm{min}$, further referred to as VVI 60 , VVI 85, VVI 110) and also VVIR. If a particular pacemaker did not allow programming in VVI to a certain rate the rate within 5 or 10 beats/min was selected-for example, for the Legend pacemaker VVI 80 was used instead of VVI 85 and for the Topaz pacemaker VVI 100 instead of VVI 110 . The rate adaptive variables for the randomised VVIR walk were programmed, based on the findings during the test walk. The patients performed two walks a day on consecutive days. The recuperation time between the tests was at least one hour. The patients did not know what programme their pacemaker was set in during the tests.

\section{STATISTICAL ANALYSIS}

Unless indicated otherwise, values are expressed as means (SD). Differences between the distance walked, the number of arrhythmias, the score on the Borg scale with the different programme settings, and heart rate in controls or rate response of dual sensor and activity sensing devices were analysed with a non-parametric signed ranks test. For individual comparison of the distance walked 
Figure 1 Top: Mean distances walked during six minute walking test related to pacing modes. $\left.{ }^{*} P<0.05\right)$. Bottom: Mean perceived levels of exertion, based on nonlinear Borg scale, related to pacing modes.
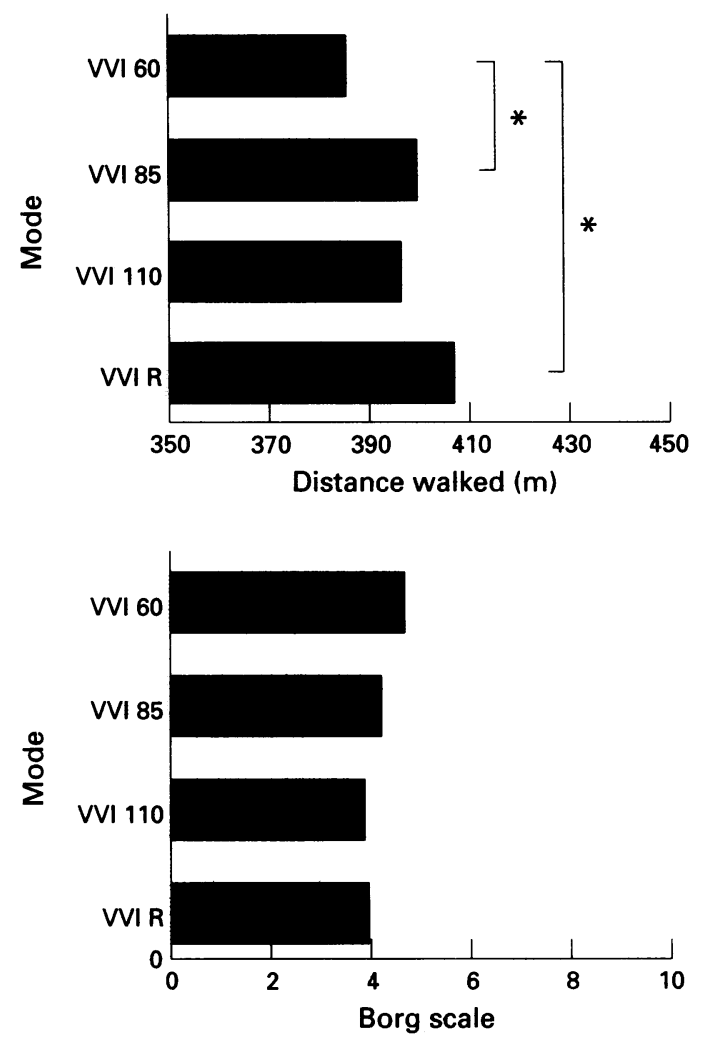

between VVI 60 and VVIR, a graduated scale was developed, with a $2.5 \%$ difference as the level of discrimination. A score of 1 was used for a difference of $2 \cdot 5-10 \%$, that of 2 for a difference of $11-20 \%$, and that of 3 for a difference of $21-30 \%$.

\section{Results}

The 13 control patients walked a mean distance of $402.0(83.4) \mathrm{m}$ during the six minutes. The mean score on the Borg scale was $4 \cdot 2(2 \cdot 3)$.

All 16 patients with pacemakers were able to walk five times in the different pacing modes during the six minutes, without interrupting the exercise test (figure 1). In the

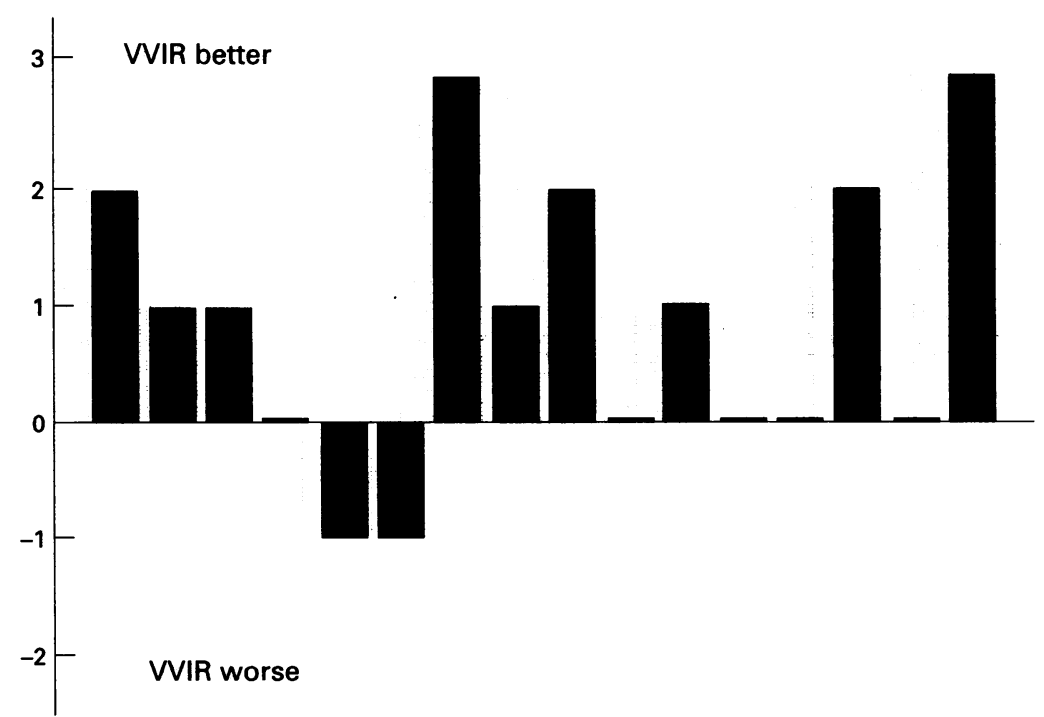

Figure 2 Graduated scale for comparing distances walked by 16 patients (see text). whole group, the distance reached in VVI 60 was 386.4 (SD 77.4) $\mathrm{m}$ and in VVI 85, 401.2 $(75.3) \mathrm{m}$; in VVI 110 patients walked 397.6 $(87 \cdot 3) \mathrm{m}$. The best performance was observed in rate responsive mode $(408 \cdot 4(69 \cdot 9) \mathrm{m})$.

The distance walked in VVI 60 was significantly shorter than that walked in VVIR (mean difference $21.9 \mathrm{~m}, 95 \%$ confidence interval 40.3 to $3.5 \mathrm{~m}$ ) and that walked in VVI $85,(14.7 \mathrm{~m}, 28.9$ to $0.6 \mathrm{~m})$. The difference between VVI 60 and VVI 110 was not significant $(11.1 \mathrm{~m}, 30.2$ to $-7.9 \mathrm{~m})$. Based on the graduated scale, only two of the 16 patients (cases 5 and 6) walked a significantly longer distance in VVI 60 than in VVIR (figure 2). The extent of exertion tended to be more pronounced in VVI 60 (mean Borg score 4.7). In VVI 85 the mean score was $4 \cdot 2$; in VVI 110 and the rate adaptive mode 3.9 (figure 1 ).

None of the patients had symptomatic arrhythmias during the exercise. The incidence of supraventricular and ventricular premature beats, sometimes in complex forms, was different between subjects. In patients with pacemakers, however, the number of premature beats remained comparable during the walks in the different pacemaker settings. The QT interval was shorter during the test in both controls and the patients with pacemakers; in the patients with pacemakers the shortening was observed in the rate adaptive and fixed rate settings. There was, however, no difference in these changes in QT interval according to VVI mode.

On Holter recording the rate adaptation of the pacemakers in the optimised VVIR setting was measured and compared with the heart rate in the controls. The programmed lower and upper rate limits were comparable in both groups of patients with devices.

In controls most of the rate increase was observed in the first two minutes of the walk; in contrast, in the activity sensor group the rate of acceleration was steep in the first minute whereas in the dual sensor group it was gradual during the first three minutes. There were significant differences between the controls and the patients with activity sensor devices at 90 seconds and between the two pacemaker groups during the second and third minute of the walk (figure 3 ). Six out of seven patients with activity sensing devices and four out of

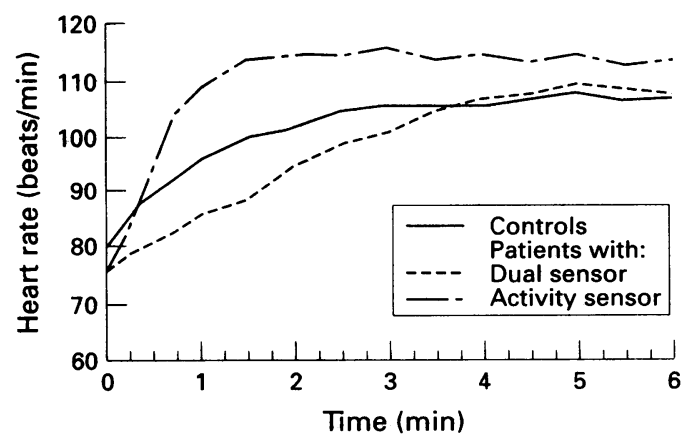

Figure 3 Acceleration in heart rate in controls and patients with activity and dual sensor pacemakers (for significant differences see text). 
Figure 4 Evaluation of activity sensing device before and after reprogramming. After the initial test walk in VVIR initial test walk in $V$ reprogrammed to a lower threshold and a higher upper rate limit, resulting in a quicker and more pronounced response of the device and the patient being able to walk further.

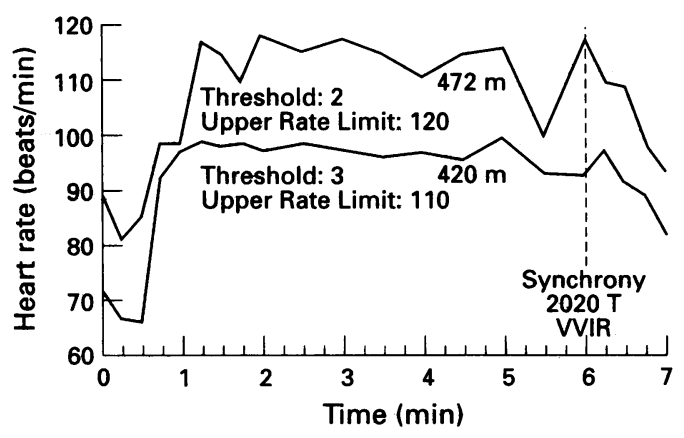

nine patients with the dual sensor pacemakers reached their upper rate limit during this walk. Furthermore, the consequences of reprogramming the pacing variables in individual patients after the initial VVIR test walk were detectable on the Holter recordings of the subsequent "randomised" VVIR walk. Figure 4 shows the rate response of an activity sensing device. After the first walk a lower threshold and a higher upper rate limit were programmed resulting in a quicker, more pronounced augmentation of the paced rate.

\section{Discussion}

Dual chamber and rate responsive pacemakers have the advantage of modulation of the stimulation rate, based respectively on a physiological impulse (the sinus node) (1-3 $^{1-3}$ an artificial sensor. ${ }^{8-10}$ To programme the pacing variables of rate adaptive devices some form of exercise test is essential. ${ }^{4-6}$ Standard exercise tests are not, however, always suitable for elderly or severely disabled patients. ${ }^{11}$ Furthermore, activity sensing devices (in contrast with devices using more physiological sensors) respond to bicycle testing only with minor rate acceleration. Body motion on a treadmill is often wooden, resulting in nonphysiological stimuli to the sensor.

Most patients with a pacemaker do not achieve the maximal predicted heart rate during daytime activities, so a maximal, symptom limited exercise test probably is not the best way to programme pacemakers. ${ }^{12}$ Furthermore, to assess physical capacity, and to appreciate whether the maximal heart rate is achieved, measurement of oxygen consumption and carbon dioxide production is desirable. ${ }^{13}$ This is another limitation for many patients. Therefore, a submaximal test is attractive, provided that it can discriminate between different settings and conditions.

The six minute walking test can give information about the exercise capacity of the patients and the rate response of the pacemakers. This form of test was first performed in patients with chronic obstructive pulmonary disease. ${ }^{14}{ }^{15}$ Hayes et al studied volunteers in a self determined casual and brisk walk in an effort to standardise the expected heart rate responses for this form of exercise. ${ }^{16}$ The duration of the walks was, however, limited to only two minutes, so the maximal heart rate for this degree of activity might not always have been achieved. Also, they studied women alone and did not give any information about associated disease. The achieved distances in two, six, and 12 minute walking tests were compared, the shorter tests being reproducible but less discriminating. ${ }^{15}$ Lipkin et al evaluated the walk in patients with mild to moderate chronic heart failure; they compared the distance covered with the maximal oxygen consumption during a treadmill test. ${ }^{17}$ The relation was curvilinear, and was most discriminating in patients with severe functional limitations. The patients preferred walking along a corridor to exercising on a treadmill. The walk was highly reproducible. ${ }^{11}$ The test is already validated in patients with pacemakers and showed a good correlation between the reached workload during bicycle ergometry and the six minute walk. ${ }^{18}$ However, there was a slightly lower performance and maximal heart rate during the walk, suggesting the submaximal character of this test. The patients were evaluated only with the pacemaker in fixed mode setting.

We studied the six minute walk in an elderly group of patients with rate responsive pacemakers because they did not have the physical ability to perform standardised exercise tests. All patients had complete heart block in order to evaluate response of the pacemaker to activity. The natural movement of the subjects during the walk allowed the rate response of the pacemakers to be studied, irrespective of which sensor it used. We found that the distance walked in the VVIR mode was longer than that walked in VVI 60 . This suggests that the six minute walk is useful in patients in whom the VVIR setting is not optimal. The good performance of patients in VVI 85 confirms this form of activity is indeed submaximal. Furthermore, differences between two sensing principles were evaluated, in comparison with the normal sinus node function in the controls. Because the six minute walk is a submaximal exercise test, we would like to see a maximal achieved pacing rate of about $75-85 \%$ of the upper rate limit. In the controls the maximal achieved rate was $73 \%$ of the maximal heart rate, corrected for age $(220$ - age). In almost all patients with an activity sensing pacemaker, however, the upper rate limit was reached after a fast onset of acceleration of the paced rate. The correlation of this paced rate with the degree of exertion was low. The dual sensor pacemakers showed a somewhat more moderate answer in the first minutes of exercise and the relation to the exertion seemed better. It is not clear, however, whether these differences were related to the characteristics of the sensor or to the algorithm in these pacemakers or whether it was influenced by our programming. The test allowed us to see the effect of reprogramming of pacemakers. Modification of the lower and upper rate limits, onset, and slope of rate acceleration and activity threshold resulted in differences of rate response.

In conclusion, we postulate that the six minute walk is a simple, short, cheap and safe submaximal exercise test that reaches the level of daily activities and is acceptable, even for 
elderly people. The test is sensitive enough to show differences of programmings of VVI and VVIR, and the rate response of different types of sensors and settings of VVIR devices can be appreciated. The good reproducibility and the natural way of moving during the walk allows repeated testing of patients with rate responsive devices to ensure an optimal programming, based on the maximal achieved pacing rate and on rate acceleration during the test.

We thank Veerle De Meyer and Pieter Depuydt for helping us and the patients during this study.

1 Perrins EJ, Morley CA, Chan SL, Sutton R. Randomised controlled trial of physiological and ventricular pacing. Br Heart f 1983;50:112-7.

2 Chu-Pak Lau. The range of sensors and algorithms used in rate adaptive cardiac pacing. PACE 1992;15:1177-211.

3 Clarke M, Sutton R, Ward D, et al. Recommendations for pacemaker prescription for symptomatic bradycardia. $B r$ Heart $71991 ; 66: 185-91$.

4 Sowton E. Exercise testing in the indication and evaluation of pacemaker treatment. Eur Heart $₹$ 1987;8:155-8.

5 Furman S. Rate-modulated pacing. Circulation 1990;82: 1081-94.

6 Sulke N, Dritsas A, Chambers J, Sowton E. Is accurate rate response programming necessary? $P A C E$ 1990;13: 1031-44.
7 Borg G. Psychophysical bases of perceived exertion. Med Sci Sports Exerc 1982;14:377-81.

8 Rossi P, Plicchi G, Canducci G, Rognoni G, Aina F. Respiration as a reliable physiological sensor for controlling cardiac pacing rate. $\mathrm{Br}$ Heart $₹$ 1984;51:7-14.

9 Humen DP, Kostuk WJ, Klein GJ. Activity-sensing, rate responsive pacing. Improvement in myocardial performance with exercise. $P A C E$ 1985;8:52-9.

10 Jordaens L, Backers J, Moerman E, Clement DL. Catecholamine levels and pacing behaviour of QT-driCatecholamine levels and pacing behaviour of QT-dri-

11 Guyatt GH, Sullivan MJ. Thompson PJ, et al. The sixminute walk: a new measure of exercise capacity in patients with chronic heart failure. Can Med Assoc $\varsubsetneqq$ 1985;132:919-22.

12 Parameshwar J, Dambrink J-HE, Sparrow J, et al. A new exercise test for the assessment of heart failure: use of a self powered treadmill. $\mathrm{Br}$ Heart f 1989;61:421-5.

13 Lipkin DP, Perrins J, Poole-Wilson PA. Respiratory gas exchange in the assessment of patients with impaired ventricular function. Br Heart $\mathcal{f}$ 1985;54:321-8.

14 McGavin CR, Gupta SP, McHardy GJR. Twelve minute walking test for assessing disability in chronic bronchitis. $B M \mathcal{F}$ 1976;i:822-3.

15 Butland RJA, Pang J, Gross ER, Woodcock AA, Geddes DM. Two, six and twelve minute walking tests in respiratory disease. $B M 7$ 1982;284:1607-8.

16 Hayes DL, Von Feldt L, Higano ST. Standardized informal exercise testing for programming rate adaptive pacemakers. PACE 1991;14:1772-6.

17 Lipkin DP, Scriven AJ, Crake T, Poole-Wilson PA. Six minute walking test for assessing exercise capacity in minute walking test for assessing exercise

18 Langenfeld $H$, Schneider B, Grimm W, et al. The sixminute walk-an adequate exercise test for pacemaker mitients. $P A C E$ 1990;13:1761-5. 